\title{
Philosophiques
}

\section{À la recherche d'une justice de structure au Nouveau-Brunswick}

\section{David Braybrooke}

Volume 3, numéro 1, avril 1976

URI : https://id.erudit.org/iderudit/203049ar

DOI : https://doi.org/10.7202/203049ar

Aller au sommaire du numéro

Éditeur(s)

Société de philosophie du Québec

ISSN

0316-2923 (imprimé)

1492-1391 (numérique)

Découvrir la revue

Citer ce document

Braybrooke, D. (1976). À la recherche d'une justice de structure au

Nouveau-Brunswick. Philosophiques, 3(1), 123-129.

https://doi.org/10.7202/203049ar d'utilisation que vous pouvez consulter en ligne.

https://apropos.erudit.org/fr/usagers/politique-dutilisation/ 
DeuXième Partie

\title{
À LA RECHERCHE D'UNE JUSTICE DE STRUCTURE AU NOUVEAU-BRUNSWICK *
}

\author{
par David Braybrooke
}

D'autres sont beaucoup mieux placés que moi pour connaittre les détails conceptuels et autres de la lutte des Acadiens au Nouveau-Brunswick pour la survivance et l'épanouissement de la culture française. De loin je sympathise avec eux, mais je ne me serais pas aventuré à commenter cette lutte si l'on ne m'avait pas demandé ce commentaire et si je ne m'étais pas jugé moi-même apte à dire quelque chose de possiblement utile sur la nature de cette lutte. Ceux qui sont plus proches que moi de cette lutte, par exemple S. Morin, sont enclins à y voir, du moins en partie, une lutte contre une injustice qui se trouve enracinée dans la structure de concepts dans laquelle la lutte elle-même a lieu. Je pense pouvoir expliquer comment peut exister une telle lutte et en même temps montrer que l'on peut espérer la résoudre, du moins par l'usage provisoire de la même structure de concepts en vue d'en construire une nouvelle.

On peut dire que tout conflit pleinement intelligible présuppose une structure commune de concepts que tous les partis en présence peuvent invoquer pour préciser trois choses:

I. L'enjeu: par exemple, possession de terres, droits touchant l'éducation, équité dans l'offre d'emploi.

II. Les raisons que l'on peut invoquer en revendiquant l'enjeu : occupation, usage immémorial, besoins humains.

III. Les moyens de pression dont disposent ceux qui ont un tel enjeu et de telles raisons.

* Réponse à l'article de S. Morin: «Sur l'injustice de structure» dans Pbilosophiques, 1 (1974), 171-192.

Qu'il me soit permis de remercier toutes les personnes qui ont collaboré avec moi à la préparation de ce texte dans sa version française actuclle, et tout particulièrement Mme Jeanne pucetti et la rídaction de la revue. 
Notons immédiatement que la «structure commune» peut être incohérente, voire même confuse, soit parce que les idées partagées d'ur: côté et de l'autre ne sont pas conséquentes, soit parce qu'il existe un certain désaccord entre les opinions reçues des deux côtés. D'ailleurs les institutions sociales peuvent imparfaitement refléter les diverses parties de la structure. Ces confusions engendrent des conflits sociaux et en aggravent beaucoup d'autres.

Ces confusions indiquées, nous ne les développerons pas, pour l'instant du moins, pas plus d'ailleurs que d'autres détails que nécessiterait une application raisonnablement nuancée de notre argumentation à la lutte des francophones au NouveauBrunswick. Notre intention est d'offrir, en guise de raisonnement fort schématique, une sorte de passe-partout capable de dénouer les énigmes d'une telle situation. C'est pourquoi nous présenterons maintenant un schéma sous forme de vision logique plutôt que véritablement historique des étapes principales de la lutte à l'égard de chacune desquelles une structure pertinemment différente de concepts se trouve en vigueur. Ce schéma rendra visible toute une gamme d'injustices :

(1) Refus par la communauté anglophone d'assimiler les francophones, tandis que les anglophones possèdent les possibilités d'emploi et se maintiennent dans une position leur permettant d'exploiter les francophones.

(2) Assimilation par la communauté anglophone, mais en même temps refus de respecter la langue française, ou de fournir des occasions pour son usage, au travail, dans les magasins, les écoles, ou dans les cours de justice.

(3) Le bilinguisme passif: la communauté accepte l'usage du français par les francophones partout où les francophones le veulent, au moins dans les situations publiques, mais la communauté anglophone ne s'engage dans aucun projet à long terme destiné à entretenir la culture francophone.

(4) La communauté anglophone apprécie la présence francophone suffisamment pour soutenir des projets efficaces, pour la préserver, pour la promouvoir et pour lui ouvrir les moyens de fournir sa contribution particulière à la vie commune de la province. 
Du point de vue des francophones, (1), (2) et (3) signifient tous des injustices, des abus systématiques contre lesquels, nous osons croire, les victimes ne pourraient pas protester à l'intérieur des structures conceptuelles et institutionnelles qui y seraient associées. Par contre, du point de vue anglophone, (1), (2) et (3) représentent chacune des étapes successives de la justice. Même l'étape (1) a pu se justifier à son époque; car il existait avant d'arriver à l'étape (1) de pires injustices ou la possibilité de telles injustices: par exemple, de propos délibéré chasser les francophones de leurs terres et en leur absence les saisir. Pour autant qu'elle implique l'absence actuelle de telles injustices, l'étape (1) est relativement juste et les anglophones peuvent se justifier de ne pas aller plus loin en affirmant qu'ils ne dérangent pas du tout les francophones qui veulent demeurer sur leur terre. .

On peut répondre naturellement qu'ils se comportent injustement vis-à-vis des francophones pour autant qu'ils refusent à ceux-ci des occasions équitables d'emploi. Si le progrès esquissé dans mon schéma se réalisait, les anglophones seraient convaincus tôt ou tard de ce fait. Nous pouvons alors imaginer que les deux communautés avanceront à l'étape (2).

Encore une fois, les anglophones estimeront qu'ils agissent suffisamment avec justice. Ils en seront peut-être convaincus encore davantage puisqu'après tout ils se souviendront qu'ils ont franchi une étape. Ils diront: "Que nous importe si ces gens veulent parler français ou faire autre chose pendant leurs loisirs? S'ils veulent des emplois et des carrières, qu'ils apprennent l'anglais; s'ils n'en veulent pas, c'est leur affaire, pas la nôtre ».

Contre cette position, les francophones plaideront que la possibilité de maintenir leur langue ne se réduit pas à une simple question de loisirs; qu'ils ont été dans le pays aussi longtemps que les anglophones, en vérité plus longtemps; que pour cette raison et pour d'autres (y compris des promesses officielles par les anglophones), ils ont le droit de se servir du français au travail, dans les magasins, les écoles, devant la justice. Pour dire de telles choses, il faut que les francophones sortent de la structure de l'étape (2); dans la mesure où ces raisonnements réussiront - et ils ont réussi jusqu'à un certain point - les deux communautés avanceront vers l'étape (3). 
Là encore, surtout en se souvenant des concessions faites dans les étapes antérieures, les anglophones tendront à penser qu'ils ont atteint le summum de la justice. Toutefois, les francophones ne seront pas satisfaits de cette justice. Ils soutiendront que le «bilinguisme» de l'étape (3) ne consiste qu'à laisser mourir la culture francophone, étant donné surtout que les injustices typiques des étapes (1) et (2) sont loin d'être tout à fait éliminées. (Seront-elles éliminées avant que l'étape (4) ne soit réalisée?)

On peut être quelque peu pessimiste au sujet de la facilité avec laquelle les anglophones accepteront cette thèse (quoique leur facilité à l'accepter ne sera pas nécessairement décisive). Le raisonnement exigc qu'ils élargissent leur conception de l'enjeu, non pas le droit de maintenir la langue française si la communauté francophone peut le faire, sans être découragée par le monde des affaires ou par le gouvernement, mais le droit à des mesures efficaces qui peuvent endiguer la dérive vers l'assimilation.

Il faut qu'un certain développement se produise simultanément dans leur mentalité à propos des raisons que l'on peut invoquer en revendiquant un enjeu de ce genre. Par exemple, l'identité culturelle ne doit pas être vue seulement comme quelque chose que les gens désirent et qu'ils doivent avoir un droit de protéger (pour autant qu'ils le peuvent), mais comme une affaire vitale que les activités passées de la communauté anglophone ont mise en danger, un danger que son inactivité actuelle a aggravé, en attendant les remèdes appropriés.

Dans la perspective des quatre étapes, on doit reconnaitre que soit de bonne volonté, soit à contrecoeur, les anglophones ont accepté la rectification des formes les plus grossières de l'injustice. Les persuader maintenant d'accepter ou de soutenir la rectification d'une forme plus subtile peut être, à cause de la limite atteinte, beaucoup plus difficile. À cet égard, la perspective est décourageante.

Décourageante aussi à l'égard d'un trait spécifique et décisif de la structure de l'étape (3). C'est un trait qui se relie aux concepts de l'item (III), où il s'agit des moyens permis de pression. L'adhésion sans réserve au principe majoritaire rend 
les anglophones aveugles à l'indignité d'une minorité ethnique contrainte de suivre une ligne de conduite non ethnique, une ligne dans laquelle elle tâcherait d'éviter toute indication que, comme minorité ethnique, elle a des intérêts qui s'opposent aux intérêts communs du reste de la population. En effet, elle se trouve alors portée à rendre son caractère ethnique invisible politiquement et peut-être aussi invisible socialement, ce qui signifie la défaite de ses aspirations culturelles par ses propres actions. Cependant, comment contester le principe majoritaire à l'intérieur de la structure héritée de concepts et d'institutions?

$\bar{A}$ d'autres égards, la perspective est plus prometteuse : car c'est le même principe de la justice qui opère pour propulser les deux communautés dans chaque mouvement d'une étape à une autre. On peut formuler le principe a peu près dans les termes suivants: Il est injuste de refuser à d'antres membres de la même société les moyens d'obtenir l'accès à des bienfaits que vous recevez et estimez vous-mêmes, du moins lorsque ces moyens étaient autrefois à leur disposition (ou à celle de leurs ancêtres) dans la même société (ou au même lieu), lorsque ces moyens ont cessé de leur être accessibles comme conséquence de vos propres actions (ou des actions do vos ancêtres), et quand ces moyens peuvent devenir disponibles de nonvean (en réalité sans aucun sacrifice substantiel de votre part).

Cet énoncé de principe est long et nous l'avons formulé en termes qui peuvent paraître excessivement ad boc. ${ }^{1}$ En effet, nous avons voulu, en le formulant, nous assurer de son application réelle au Nouveau-Brunswick. Toutefois, les réserves qui sont faites dans cette formulation sont loin d'être simplement ad boc. Elle reflètent des considérations qui figuraient dans toute appréciation exacte de la situation au Nouveau-Brunswick, selon les principes fondamentaux de la justice. De plus, - un autre aspect du même problème, - ces réserves ainsi formulées rendent intelligibles aux anglophones, en des termes que ceux-ci sont prêts à reconnaître, au moins dans leurs moments raisonnables, à la fois la nature de l'injustice en jeu (s'arrêtant avant la spécification des bienfaits relatifs à chaque étape) et leur responsabilité d's̀ remédier.

Le mouvement d'une étape à une autre dans la perspective définie par la gamme d'injustices peut être vu comme le 
mouvement d'une structure de conflit plus étroite à une structure plus large. Étant donné que les anglophones n'ont pas avancé plus loin que l'étape (3), revendiquer des enjeux qui ne seront pleinement intelligibles que lorsque les termes de l'étape (4) auront été acceptés par tous et y juxtaposer des raisonnements eux-mêmes non encore intelligibles, c'est employer des arguments qui s'étendent au-delà de la structure actuelle. Toutefois, si nous avons raison de donner au principe de la justice que nous avons formulé le rôle que nous lui avons donné, il opère comme un principe avec lequel on peut contester n'importe quelle structure actuelle. C'est un principe qui est aussi paradoxalement accessible comme une partie de la structure actuelle elle-même. Les faits auraient été différents. Cependant, la structure totale de concepts et d'institutions qui est pertinente aux conflits sociaux et politiques du Nouveau-Brunswick - une structure partagée par les anglophones aussi bien que par les francophones - contient en fait des éléments qui, comme ce principe, peuvent servir la cause de la réforme structurelle.

Je pense qu'il ne faut pas non plus abandonner l'espoir quant à l'application du principe majoritaire. Là encore, nous voyons une structure de concepts er d'institutions qui est assez compliquée et indéterminée. Là encore, nous voyons une question d'injustice de structure que l'on doit nuancer. On peut trouver l'injustice de structure en regardant l'usage actuellement prédominant, un usage qui contrecarre justement la considération de n'importe quel aspect de cette injustice. On peut en même temps trouver dans la structure, en observant la capacité conceptuellement spécifique de celle-ci de se transformer, des éléments qui favorisent les changements progressifs. Je pense que l'on peut persuader les champions du principe majoritaire que, dans leur adhésion sans réserve, ils n'ont pas prévu l'application régulière de ce principe contre les intérêts d'une minorité permanente. On peut les persuader que faire ceci est employer le principe aux dépens de la minorité, donc l'employer en opposition à leur propre sens de la justice. ${ }^{*}$ Il y aura encore beaucoup à faire lorsque les anglophones seront persuadés de ce point. On ne peut pas facilement prévoir quels moyens

* Cf. ma discussion de "The reassortment condition» dans Three Tests for Democracy: Personal Rigbts, Human Welfare, Collective Preference (New York: Random House, 1968), pp. 170-172. 
institutionnels peuvent contrebalancer, avec les effets désirés, l'opération du principe majoritaire. Toutefois, l'on connaît de tels moyens - par exemple, systèmes de droits retranchés, autonomie en ce qui concerne la politique de l'éducation, etc. En tout cas, reconnaître le point et le principe de justice formulés plus haut peut établir les prérequis pour échapper à une condition d'injustice de structure et à la fois avancer vers une condition dans laquelle les ajustements structuraux facilitent la disparition des injustices enfin reconnues.

\section{Dalhousie University}

1. Les termes utilisés pcuvent également paraître manquer de prudence. En effet, n'est-il pas possible d'invoquer un contre-exemple qui montrerait que ces autres membres de la société, ou leurs ancêtres, se sont délibérément bloquer, suite à une action consciente de leur part, l'accès à certains bienfaits? Mais on peut difficilement croire que ce soit le cas au NouveauBrunswick. En plus, on peut mettre en doute que les gens aient le pouvoir moral de se priver de choses essentielles pour vivte une vie honnête et heureuse, ou d'en priver leurs descendants. Cette question appelle une réponse du même type que celle de Rousseau quand il se demande si un homme a le droit d'emmener ses enfants en esclavage (cf. Du contrat social, livre I, chapitre IV). 